\title{
Empirical Size Corrections for Shadowgraph Imaging of Non-Spherical Droplets
}

\author{
C.M. Sipperley*1, K.M. Bade ${ }^{2}$, P.W. Vesely ${ }^{2}$, R.J. Schick ${ }^{2}$ \\ ${ }^{1}$ Step 2 Consulting, Inc., Palm City, FL 34990, USA \\ ${ }^{2}$ Spraying Systems Co., Wheaton, IL 60187, USA \\ *Corresponding author email: chad@step2inc.com
}

\begin{abstract}
Droplet sizing by shadowgraph imaging is a classic and direct method for evaluating the size of droplets or particles. However, liquid droplets are often non-spherical due to surface oscillations generated at breakup, aerodynamic drag forces, or through other mechanisms that overcome internal surface tension forces. Multi-perspective imaging of droplets allows greater understanding of non-spherical droplet shapes, when to expect non-spherical droplets, and potential corrections to better capture the effective diameter and volume contained within the droplets. The forces acting upon the droplets are related to non-dimensional parameters such as the Weber and Ohnesorge numbers, and empirical corrections are developed and evaluated for their ability to account for single-perspective shadowgraph images. In this work the authors demonstrate a technique for determining a most-probable geometry for highly-distorted fluid parcels (blobs) as imaged from multiple views. The basis of the technique is the assumption that surface energy should be minimized for the most probable three-dimensional shape consistent with the multiple images. Once a fully-resolved three dimensional shape of a fluid blob has been determined the authors demonstrate how this information could be used as a correction factor for single images of similarly distorted blobs.
\end{abstract}

\section{Keywords}

Large Droplets, Blobs, Non-Spherical Droplets, Shadowgraph Imaging, 3D

\section{Introduction}

There are several techniques for measuring the size of droplets in sprays including laser diffraction, phase Doppler particle interferometry, and shadowgraph imaging. All techniques have their advantages and disadvantages and have ideal applications. Droplet sizing via shadowgraph imaging is ideal for sprays containing non-spherical droplets. Non-spherical droplets are common in effervescent sprays where bubbles of gas may be present inside of the droplets [1]. Non-spherical droplets may also be present in high velocity sprays like in paint spraying with rotary bell atomizers [2] and in combustion [3]. In this work, the authors were motivated to characterize rain simulation sprays composed of large drops (in the millimeters range) that remained highly non-spherical due to residual breakup oscillations and internal flow.

A challenge with measuring droplet size using the shadowgraph technique is oversizing the droplet. This is typically thought of in terms of out of focus droplets which appear larger than they are when in focus. This has been studied thoroughly and well defined techniques have been established to prevent over-sizing out of focus droplets including by Lee and Kim [4], Blaisot [3], Rydblom [5], and others.

In this investigation, an exploration of over-sizing of non-spherical droplets from the analysis of a single-perspective shadowgraph image is explored. An experiment was conducted to obtain three perspective shadowgraph images of a non-spherical droplet. These images were used to construct a three-dimensional representation of the non-spherical droplet using a minimum surface energy approach. This representation of the non-spherical droplet was then used to calculate the effective diameter from many different perspectives to generate the possibilities 
of perimeter vs area that could be obtained from the droplet sizing using single perspective shadowgraph imaging.

\section{Experimental Setup}

To obtain the the data needed for this diagnostic technique investigation, an experiment was constructed to acquire images from large blobs of water using three cameras. This experimental setup is shown in Figure 1. A single nozzle was centered above the axis of the array of cameras. Large blobs and droplets were generated using a large, straight orifice and a near zero pressure drop across the orifice.

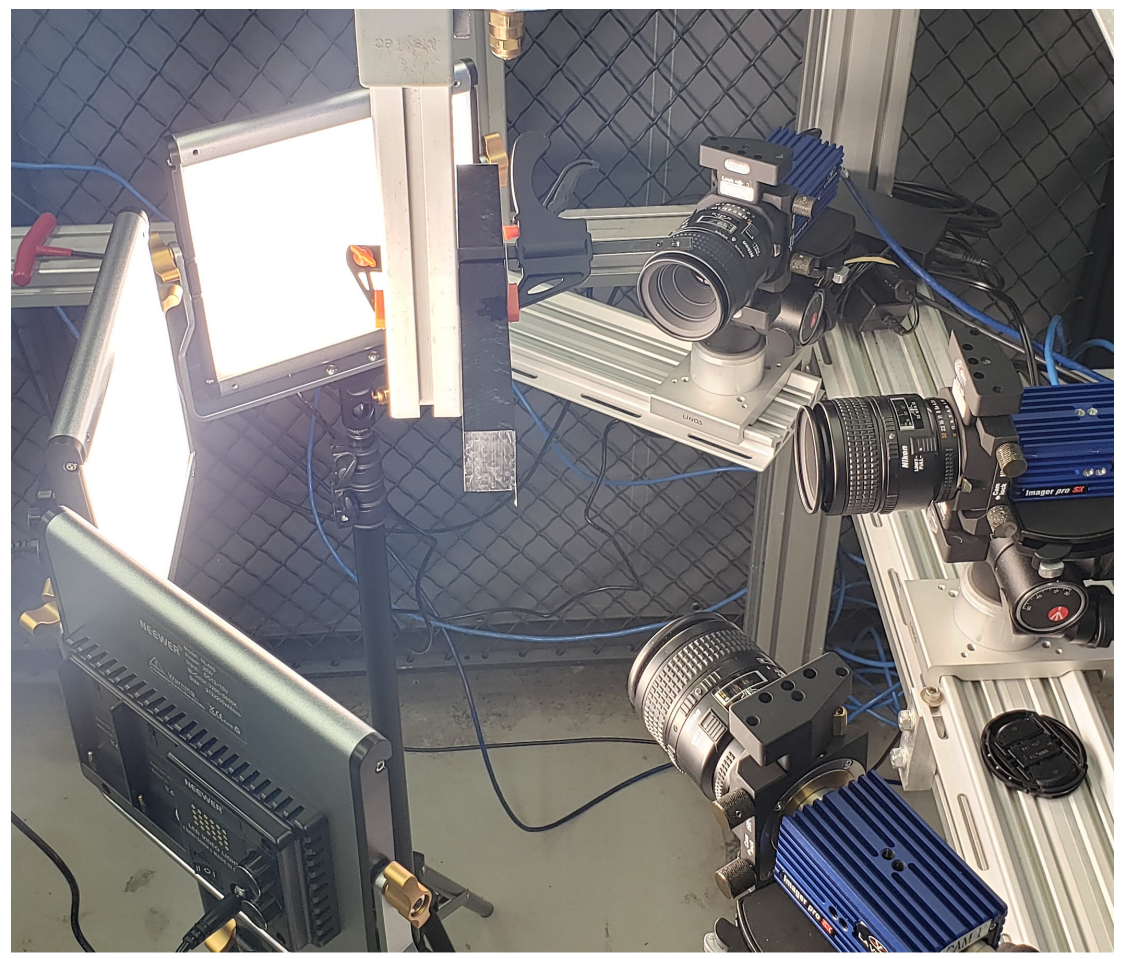

Figure 1. Full experimental setup.

A LaVision GmbH FlowMaster Tomographic PIV system was used to acquire synchronized images from three cameras for this experiment. The system was operated using the DaVis 10 software and the camera triggering was conducted using the LaVision PTU X programmable timing unit. The cameras were outfitted with Nikon AF Micro-NIKKOR $60 \mathrm{~mm}$ f/2.8D lenses. Imager Pro SX 5M cameras were used, which have the following specifications:

- Sensor size: $8.47 \times 7.10 \mathrm{~mm}$

- Resolution: 2448 x 2050 pixels

- Pixel size: $3.45 \times 3.45 \mu \mathrm{m}$

The cameras were each fixed to three-axis tripod heads that were then mounted to optical rails. The cameras were positioned 60 degrees from each other with the center of the tripod mounts on a $800 \mathrm{~mm}$ diameter arc, as is shown in Figure 2. This resulted in an approximate focal plane distance of $240 \mathrm{~mm}$ for each camera. With the use of the $60 \mathrm{~mm}$ fixed focal length lenses, this resulted in a field of view of $25.2 \mathrm{~mm} \times 21.0 \mathrm{~mm}$. A scale of known distance between marks was used to calibrate the image of each camera.

Back-illumination lighting was provided with three Neewer Model NL480 lights positioned opposite and normal to each camera as can be seen in Figure 1. Each light provided $28 \mathrm{~W}$ 
power with an overall size of $212 \times 200 \mathrm{~mm}$, and was composed of two-color LEDs at $3200 \mathrm{~K}$ $5600 \mathrm{~K}$, with 24 columns $(\mathrm{dx}=7.95 \mathrm{~mm})$ and 20 rows $(\mathrm{dy}=7.85 \mathrm{~mm})$, with $3360 \mathrm{Lux} / \mathrm{m}$. Each individual LED is estimated to be $3.8 \mathrm{~mm}$ in diameter.

The three axes for the lab-space coordinate system are defined relative to the center camera. The $x$-axis is the horizontal axis as seen in the image (positive to the right). The $y$-axis is defined by the optical axis of the lens for the center camera (Cam 1, with positive away from the camera). The z-axis satisfies a right-hand coordinate system - the up-down axis in the image plane with up being the positive direction. In this coordinate system, the outer two cameras have been rotated about the z-axis such that this axis is in sharp focus for all three imaging systems.

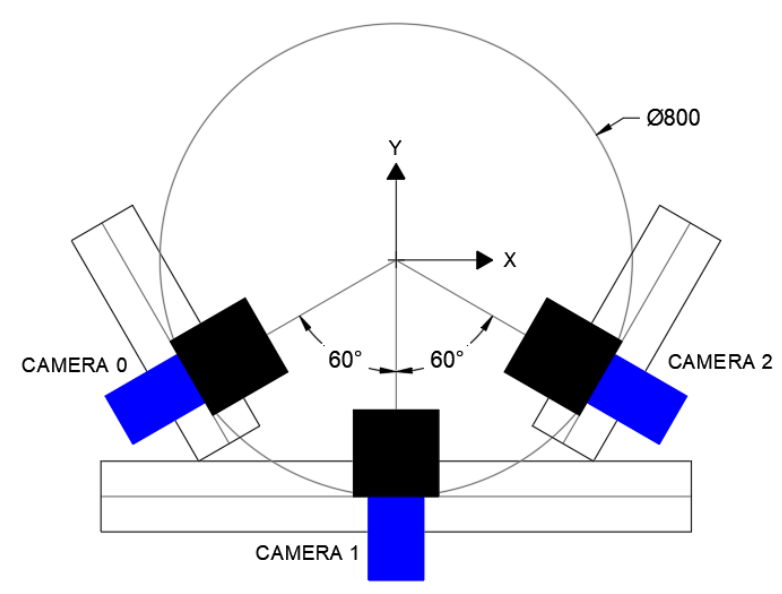

Figure 2. Camera placement schematic.

\section{Results and discussion}

Figure 3 presents the images acquired simultaneously from the three cameras of non-spherical drop of water, or 'blob'. The droplet edges are sharp and clearly defined relative to the blurred background, while the grid-pattern of each back-illumination LED panel can be seen refracting through the center of the droplet from each perspective.
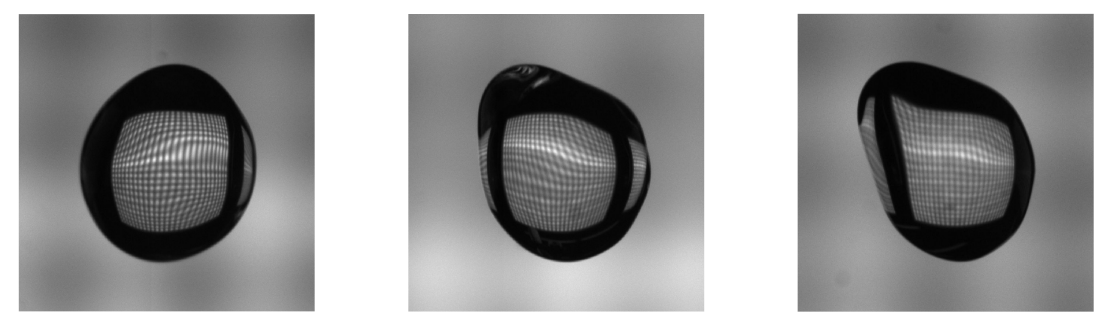

Figure 3. Simultaneous images of the droplet from three camera perspectives. From left to right they are camera $0,1,2$. The LED grids used for backlighting are visible in the images as refracted by the droplets.

In each image, the outline of the blob image represents a silhouette of the blob from that view. Any resolved object must match these three silhouettes. The maximum-volume object that meets this requirement, shown in Figure 4 would have hexagonal cross sections at every plane perpendicular to the z-axis.

While satisfactory to accommodate the 3-perspective camera images, the hexagonal object in Figure 4 is not a shape that will exist natural gas and liquid forces; most importantly here, surface tension. There are three constraints that must be taken into account to determine the fully-resolved three-dimensional shape of a liquid blob from a finite number of images: silhouette matching, surface energy, and edge tangency. These constraints are explored further 

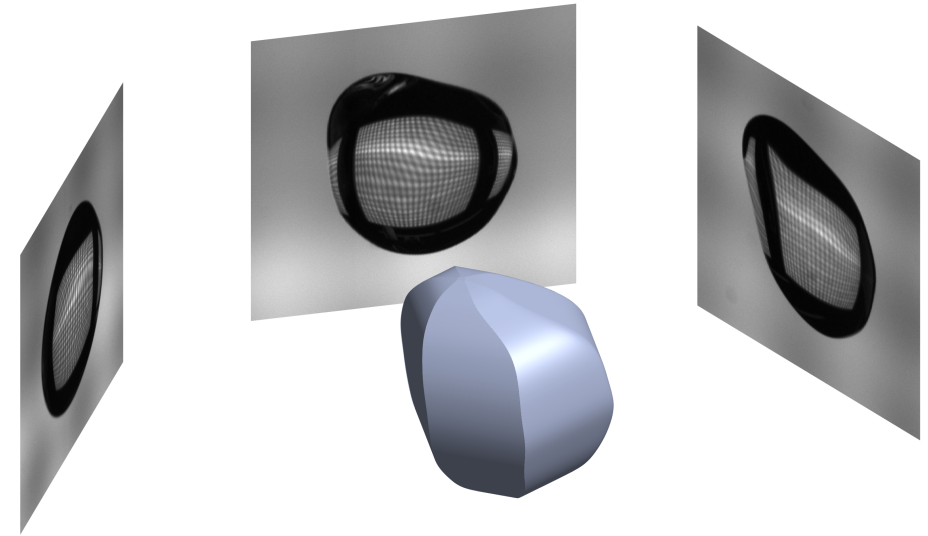

Figure 4. The three images of the droplet are placed in a three-dimensional plot parallel to the respective camera. The edge of the drop in each image represents the boundary of a silhouette or projection along that axis. With a finite number of images there exist an infinite number of three-dimensional shapes that would generate the recorded silhouettes. The object rendered in the center of this figure is the maximum-volume shape that satisfies the silhouette requirement.

in order to allow a superior 3-dimensional droplets to be recovered from the limited number of camera views.

The maximum-volume object would be physically unrealizable owing to the surface energy contained in the sharp corners. Due to surface energy considerations, the surface of a liquid blob must be infinitely differentiable. Minimizing the surface energy of a liquid blob leads to minimizing the surface area. Absent knowledge of other energy modes, the most probable fullyresolved shape will be the one with minimum surface area while satisfying all other constraints.

The infinite-differentiability and silhouette constraints lead to a final constraint. The surface of the resolved object must be tangent to the optical axis direction vector for each camera for every point projected into the outline of the silhouette.

The procedure for finding the most-probable blob surface is as follows. A finite number of evenly-spaced planes perpendicular to the z-axis are defined. At each plane, a closed analytic curve representing the perimeter of the cross section is created. The nature of the analytic curve ensures that the silhouette and tangency requirements are always met. Each analytic curve has several free parameters (17 in this work) and there are many such planes (typically several dozen). The free parameters are then optimized to minimize the surface-area-to-volume ratio. Strictly speaking, the (surface area) $)^{3} /$ (volume) $)^{2}$ is the parameter minimized here, because the volume of the blob is a function of the chosen free parameters and the surface area cannot be minimized alone. The final shape is the lofting from one perimeter to the next.

Consider one cross-sectional plane as described above, as shown in Figure 5a. The silhouette constraint creates a hexagonal region that entirely encompasses the perimeter. The tangency constraint requires that the perimeter must touch this hexagon at one point on each side and that it parallel to that face at that point, see the control points ('knots') and tangency lines in Figure $5 \mathrm{~b}$ which illustrates the cross section taken from Figure $5 \mathrm{a}$. The dashed lines are the silhouette locations from each of the three images at this plane. The corners of the hexagon formed from these lines are six of the knot locations for the NURBS curve representing the cross section of the blob at this plane. The other six knot locations are placed between these corner points; one per hexagon edge. The locations of these mid-edge knots are allowed to vary and are a free variable during optimization. The relationship of one middle point $\left(M_{i}\right)$ and the two corner points $\left(C_{\text {near }}, C_{\mathrm{far}}\right)$ for one edge are demonstrated in the figure. As described in Equation the location of $M_{i}$ can vary from $C_{\text {near }}$ to $C_{\text {far }}$ (as viewed from Cam 0). Optimization of the weights pulls the curve off the corner knots but the order of the NURBS curve and three linearly-aligned knots ensures the NURBS curve always passes through each midpoint knot.

The technique can properly account for concave surfaces but only when each cross section 


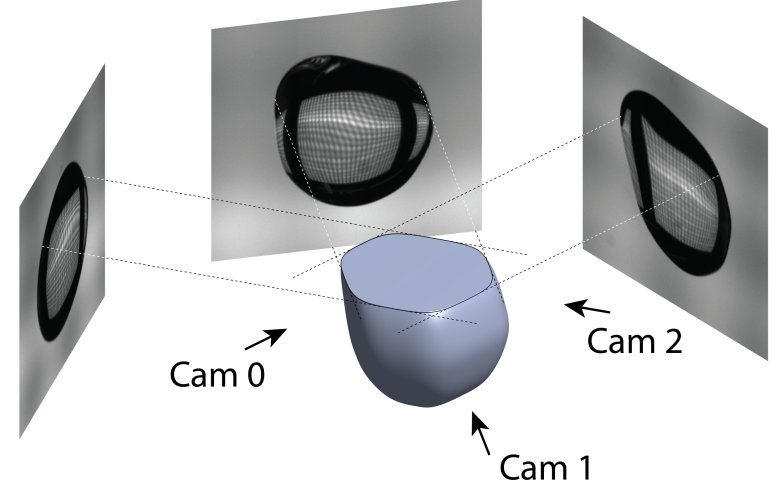

(a) Single Plane Slice

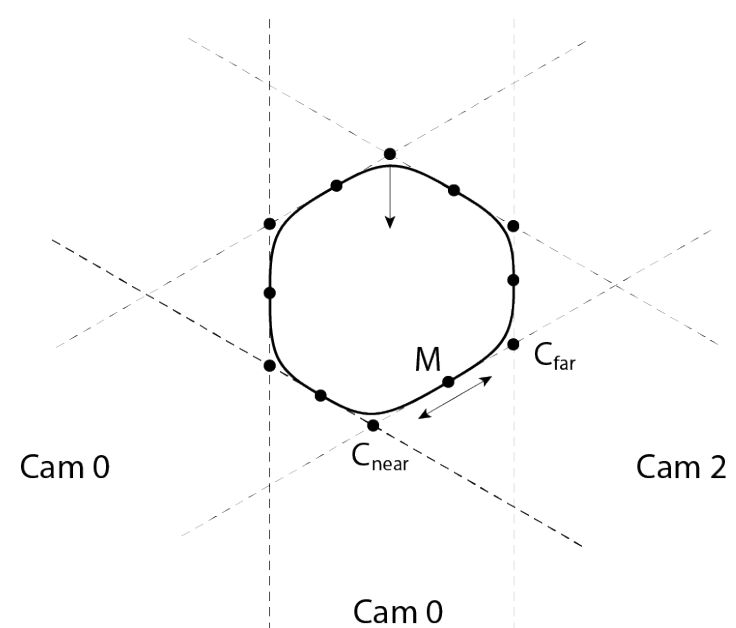

(b) Knots and tangency lines

Figure 5. An illustration of the construction of the blob geometry by means of a series of NURBS curves at each $z$ plane. An initial geometry is constructed by considering the cross section of the blob one plane at a time. As shown in (a), a series of NURBS curves are placed in space that satisfy the silhouette requirements. Working from bottom-to-top, rendering has been halted at a plane to highlight the process. The edges of the images at that height are determined and lines perpendicular to the image at that location are cast. A view of this plane from above is shown in (b). The corners of the hexagon formed from these lines are six of the knot locations for the NURBS curve representing the cross section of the blob at this plane. The other six knot locations are placed between these corner points; one per hexagon edge. The locations of these mid-edge knots are allowed to vary and are a free variable during optimization. The relationship of one middle point and the two corner points for one edge are demonstrated in the figure. As described in Eqn 1, the location of $\mathrm{M}$ can vary from Cfar to Cnear (as

viewed from camera 0). Optimization of the weights pulls the curve off the corner knots but the order of the NURBS curve and three linearly-aligned knots ensure the NURBS curve always passes through each midpoint knot.

perpendicular to the z-axis is convex; which is a planned future application of the broader effort. The authors chose a fourth-order NURBS curve representation of the perimeter to naturally satisfy these requirements but recognize that this choice is not unique. NURBS were selected over a polynomial description because the former naturally exhibits a convex hull property that ensures the curve will never exit the control hexagon. B-Splines and Bézier curves also exhibit the convex hull property but cannot identically represent a conic section such as a circle.

A NURBS curve is defined by its order, knot vector (values and locations), and weight vector. A fourth-order NURBS curve was chosen to maintain $\mathrm{G}^{2}$ (curvature) continuity around the curve. The locations of the knots were chosen to represent the control hexagon. The silhouette constraint requires the curve to touch the control hexagon somewhere on each face. That suggests there should exist at least one knot location on each face. Ordinarily to ensure a NURBS curve passes through a location in space, a knot value is repeated. However, this reduces the continuity order and does not address the direction aspect of the tangency requirement. To simultaneously satisfy the positional and tangency continuity requirements, three knots are place on each hexagonal face. That ensures that the NURBS curve passes through the middle of the three knots and is tangent to the control hexagon at that location. Such an approach leads to the possibility of having 18 knot locations - three on each of six faces. However, if six of the knots are chosen as the corners of the control hexagon the number of knots can be reduced to 12. The final values for the knot vector were chosen to be evenly spaced. While varying these values can alter the curve, the added number of variables for minimization did not justify the additional flexibility - especially when moving the location of the tangency points accomplishes a very similar adjustment.

The values of scaling factor $m_{i}$ can vary from 0 to 1 such that the location of the midpoint, $M_{i}$, is defined as

$$
M_{i}=m_{i} C_{\mathrm{far}}+\left(1-m_{i}\right) C_{\text {near }}
$$

where $C_{\mathrm{far}}$ and $C_{\text {near }}$ are the locations of the corner points furthest-from and closest-to the 
camera, respectively. To maintain the convex hull property for NURBS curves, the weight vector must be non-negative. Additionally, the absolute values are not important; only the relative values affect the shape. As such, the values were also limited to a range of 0 to 1 .

Rather than enforce a range limit on the variables during optimization, a transformation function was chosen that would allow variables to scale from $-\infty$ to $\infty$ during optimization that would result in values ranging from 0 to 1 for $m_{i}$ and the weights. The function chosen is

$$
k_{i}=0.5+0.5 \tanh \left(3 r_{i}\right)
$$

where $k_{i}$ is any scaled value and $r_{i}$ is the value altered during minimization.

Following this process, the hexagonal object presented in Figure 4, defined purely by the silhouette image borders, was corrected to minimize the surface energy through minimization of the surface-area-to-volume ratio. The resulting corrected three dimensional object is provided in Figure 6.
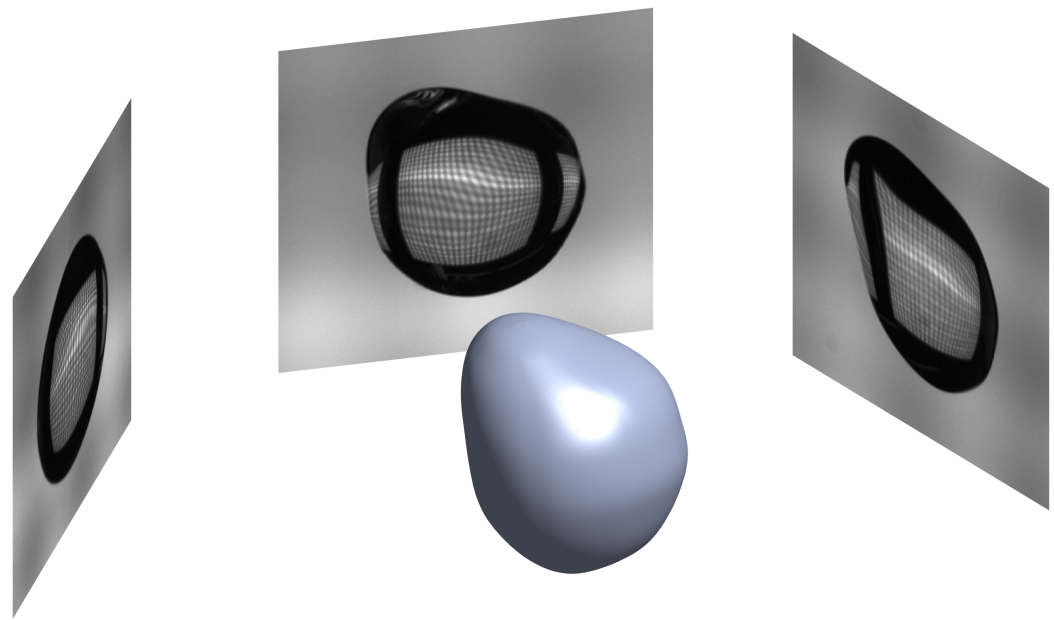

Figure 6. The result of the surface energy minimization process results in a fully-resolved representation of the blob.

With the recovery of a three dimensional droplet which satisfies the physical requirement of minimum surface energy (minimum surface-area-to-volume ratio), additional interrogations may be conducted using the 3D shape. As the motivation of this work is to determine the volume distribution of the drops, a volume-equivalent diameter will be considered the "actual" diameter of the blob. In addition, we can consider how such a blob would appear as imaged from any orientation. Figure 7 presents six views of the recovered 3D droplet.

Extending this rotation/orientation exercise, and extracting the perimeter over 2562 perspectives, a trend of droplet characteristic perimeter to area can be found and is plotted in Figure 8a; where $P^{*}$ is the perimeter of the rotated blob normalized by the volume-equivalent diameter of the solved blob, $D_{\text {actual }}$, and $A^{*}$ is the area of the rotated blob normalized by $D_{\text {actual }}^{2}$. For each view, the hydraulic diameter, $D_{\text {hyd }}=4 \mathrm{P} / \mathrm{A}$, is compared to the actual volume-equivalent diameter. Such a distribution built for a large ensemble of droplets would allow the creation of a correction factor to unbias single images of similarly-distorted blobs.

The authors are aware of the need to validate the performance of this technique with images of fluid blobs of known size. Unfortunately, the authors are unaware of any diagnostic technique that could provide a better estimate of volume than the work proposed here. Rigid objects of known-volume could be imaged and analyzed, but such an object does not necessarily represent a geometry that is physically realizable by a fluid blob subject to aerodynamic, inertial, and surface-tension effects. Such a comparison would simply be evaluating whether the target object is compatible with this analysis rather than determining if this technique is appropriate to characterize liquid blobs. Ultimately, evaluation of the performance of this technique will likely 

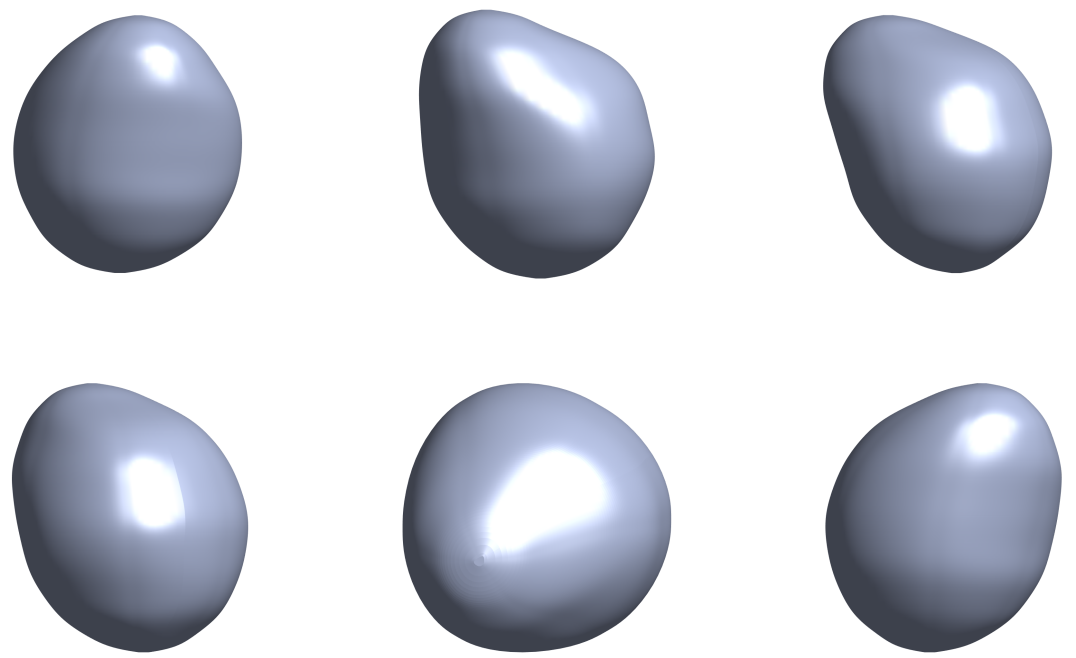

Figure 7. The optimized blob from Figure 6 from six different views. In the top row, from left to right, the views match those of cameras 0,1 , and 2 respectively. These figures are directly comparable to the raw images in Figure 3 . In the bottom row the blob is viewed from the left, top, and right relative to camera 1.

require comparison to numerical atomization models. Until such work has been performed, the authors present this technique as a work-in-progress with the argument that the results appear reasonable and that the difference in the maximum-volume solution (Figure 4) and the final optimized geometry (Figure 7) is far smaller than that which can come from any given uncorrected two-dimensional image as suggested by Figure $8 \mathrm{~b}$.

\section{Conclusions}

In this work the authors have demonstrated a method for generating a most-probable, fullyresolved geometry of a non-spherical droplet as viewed from three cameras simultaneously. A series of NURBS curves representing the cross sections of the drop are optimized to minimize the surface energy of a shape consistent with the three projected views. Such a fully-resolved geometry can be manipulated and interrogated for additional information. Additional projections from different views were considered as is this droplet were being imaged from many orientations and the effect of such a rotation was shown. In addition, the hydraulic diameters of each of those projections was compared to volume-equivalent diameter of the fully-resolved geometry.

In the future, the authors plan to apply this technique to a large ensemble of droplets in a rain simulation spray to obtain a better volume distribution measurement of the drops generated. This may include utilization of the LED grid in the background with is refracted through the droplets to better inform on concave droplet curvature. Expanding the process to identify and independently evaluate multiple drops in view. And most importantly, moving the correction methods to single-camera based corrections using preliminary knowledge of the spray from multiple views.

\section{Acknowledgments}

The authors would like to acknowledge and thank Daniel Belknap of Spraying Systems Co. for his assistance in setting up and acquiring images for this experiment.

\section{Nomenclature}

C Corner Point

$M \quad$ Mid Point 


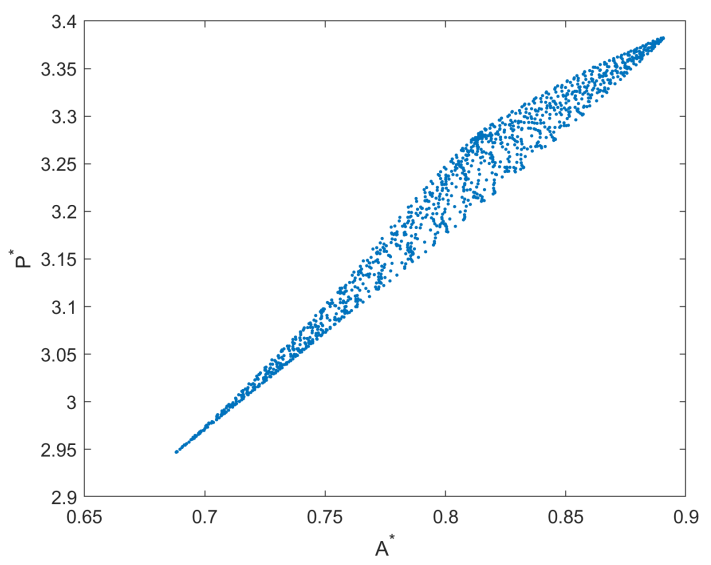

(a) Normalized Droplet Area to Perimeter

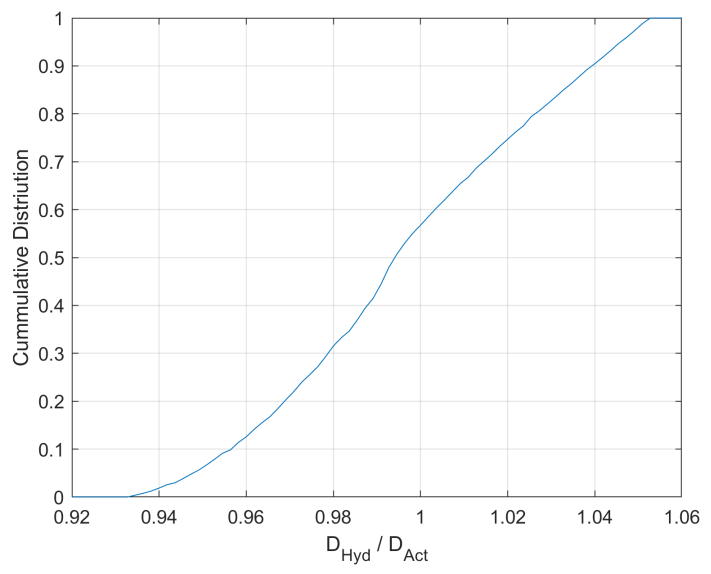

(b) Diameter ratio distribution

Figure 8. The projected area and perimeter of the blob is a function of the view vector. In (a) the normalized perimeter vs. area for many such views are plotted. The exact values are unimportant as the underlying object does not have a spherical shape. However, the scatter of these points highlights how much a single object's apparent parameters may change depending on orientation. In (b), the hydraulic diameter of each view is compared to the volume-equivalent diameter of the optimized shape from Figure 6.

$\begin{array}{ll}m & \text { scaling factor } \\ k & \text { scaling factor } \\ r & \text { scaling factor } \\ i & \text { point index }\end{array}$

\section{References}

[1] Gomez, J., Fleck, B., Olfert, J., McMillan, J., 2010, ILASS - Europe 2010, 23rd Annual Conference on Liquid Atomization and Spray Systems, Brno, Czech Republic.

[2] Wilson, J.E., Grib, S.W., Ahmad, A.D., Renfro, M.W., Adams, S.A., Salaimeh, A.A., 2018, Coatings, Vol 8,174, pp. 1-17.

[3] Blaisot, J.B., Yon, J., 2005, Experiments in Fluids, Vol 39, pp. 977-994.

[4] Lee, S.Y., Kim, Y.D., 2004, KSME International Journal, Vol 18:6, pp. 879-894.

[5] Rydblom, S., Thörnberg, B., 2016, IEEE Sensors Journal, Vol 16:8, pp. 2714-2725.

[6] Gagarinov, P., Spheretri https://github.com/pgagarinov/spheretri, GitHub, Retrieved March 26, 2021.

[7] The MathWorks, Inc., MATLAB 2019a fminsearch, https ://www . mathworks.com/help/matlab/ref/fminsearch.html\#References. 